Borys M. Kwinta ${ }^{1}$, Roger M. Krzyżewski ${ }^{1}$, Kornelia Kliś ${ }^{1}$, Ewelina Grzywna ${ }^{1}$, Krzysztof Stachura ${ }^{1}$, Dariusz Adamek ${ }^{2}$

\title{
The reliability of the specimens for neuropathological evaluation in pituitary adenomas treated via transphenoidal route
}

\author{
Corresponding author: \\ Borys M. Kwinta, PhD \\ Department of Neurosurgery \\ and Neurotraumatology, Jagiellonian \\ University Medical College, \\ Botaniczna 3 St. \\ 31-501 Kraków, Poland \\ e-mail: bmkwinta@gmail.com
}

Medical Research Journal 2018;

Volume 3, Number 1, 23-27

10.5603/MRJ.2018.0004

Copyright (C) 2018 Via Medica

ISSN 2451-2591

\begin{abstract}
ABSTARCT
Introduction: There are some discrepancies between immunohistochemical staining results and clinical picture of pituitary adenomas. Such a discordance may be caused by multiple factors. The problem of securing the histopathological material during the transsphenoidal pituitary surgery and its reliability for neuropathological evaluation deserves a special attention. The surgical biopsy does not always meet the criteria required for a immunohistochemical staining and sometimes not even for the routine histopathological examination.

Aims: To determine the frequency of unreliable material for histopathological examination and factors influencing the reliability of histopathological specimens after surgery for pituitary adenomas.

Material and methods. The hematoxylin and eosin sections were examined in detail. with a special attention to the presence of incidental findings, i.e. admixture of normal pituitary gland tissue, signs of hemorrhage, necrosis, thermal artifacts, inflammatory changes, respiratory epithelium, vessels or cholesterol granuloma. The impact of incidental findings on further immunohistochemical analysis was investigated. The relationship between the magnetic resonance imaging (MRI)- determined tumor size and the area of histopathological specimen was assessed and considered as a reliability parameter.

Results: The unreliable material was estimated at the level of $11.8 \%$. It was assessed that hemorrhages, neurohypophysis, necrosis and quantity of collected histopathological material had the statistically significant impact on the reliability of the histopathological material. The statistical analysis did not show any relation between the reliability of the histopathological material and the MRI-determined volume of the tumor. Conclusions: The presence of some additional tissue elements and artifacts in the histopathological specimen makes the immunohistochemical evaluation difficult or even impossible. However, this problem is related to a relatively low percentage of cases, mainly small tumors.

Key words: transsphenoidal pituitary surgery, histopathological specimen, reliability
\end{abstract}

Med Res J 2018; 3 (1): 23-27

\section{Introduction}

The routine diagnostics of the pituitary tumors is complex. It is based on clinical, endocrinological, radiological and neuropathological evaluation [1-3].

The histopathological diagnosis of removed adenoma is crucial for further treatment and prognosis [4-6]. By analyzing histopathological features we are able to predict the possible clinical course of pituitary adenomas [7-8]. Neuropathological examination of the specimen obtained from the material collected during the surgery relays, except routine hematoxylin and eosin (H\&E) staining, on series of the immunohistochemical examinations as well as on ultrastructural evaluation [9-13].

For the pituitary tumors diagnostics, a very important issue is the reliability of the histopathological specimen. The specimen is considered reliable if allows to determine the histopathological diagnosis, regardless of the specimen size. It means that from the formalin embedded tumor we have to obtain at least eight specimens (H\&E, immunohistochemical stainings for anterior lobe hormones and alpha-subunit). However, 
the histopathological material is not always collected in sufficient amount during the surgery. It may result from the size of the tumor itself. Theoretically, the greater tumor we treat the greater material should be obtained

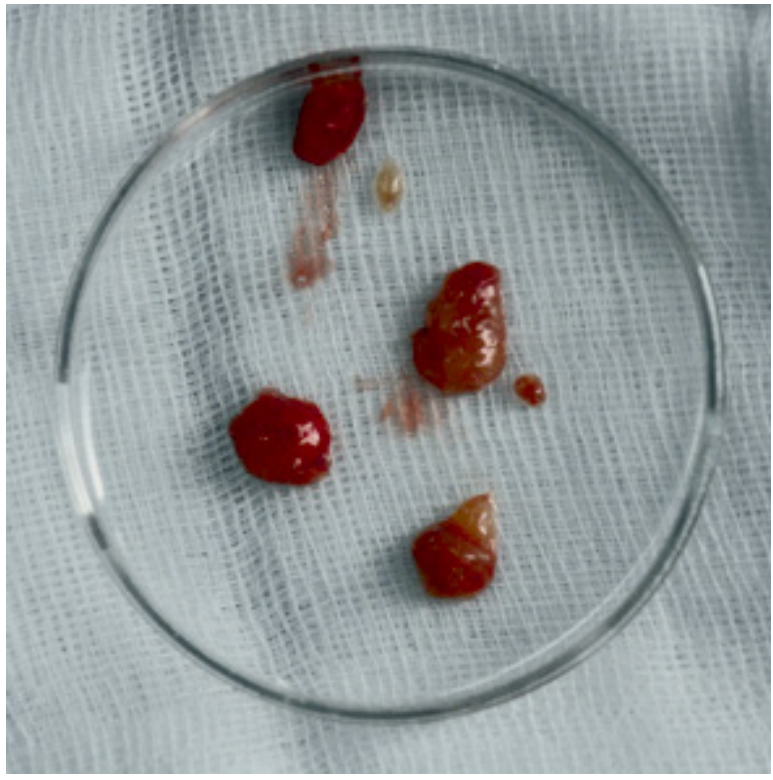

Figure 1. Specimen obtained during transspehenoidal surgery for pituitary adenoma
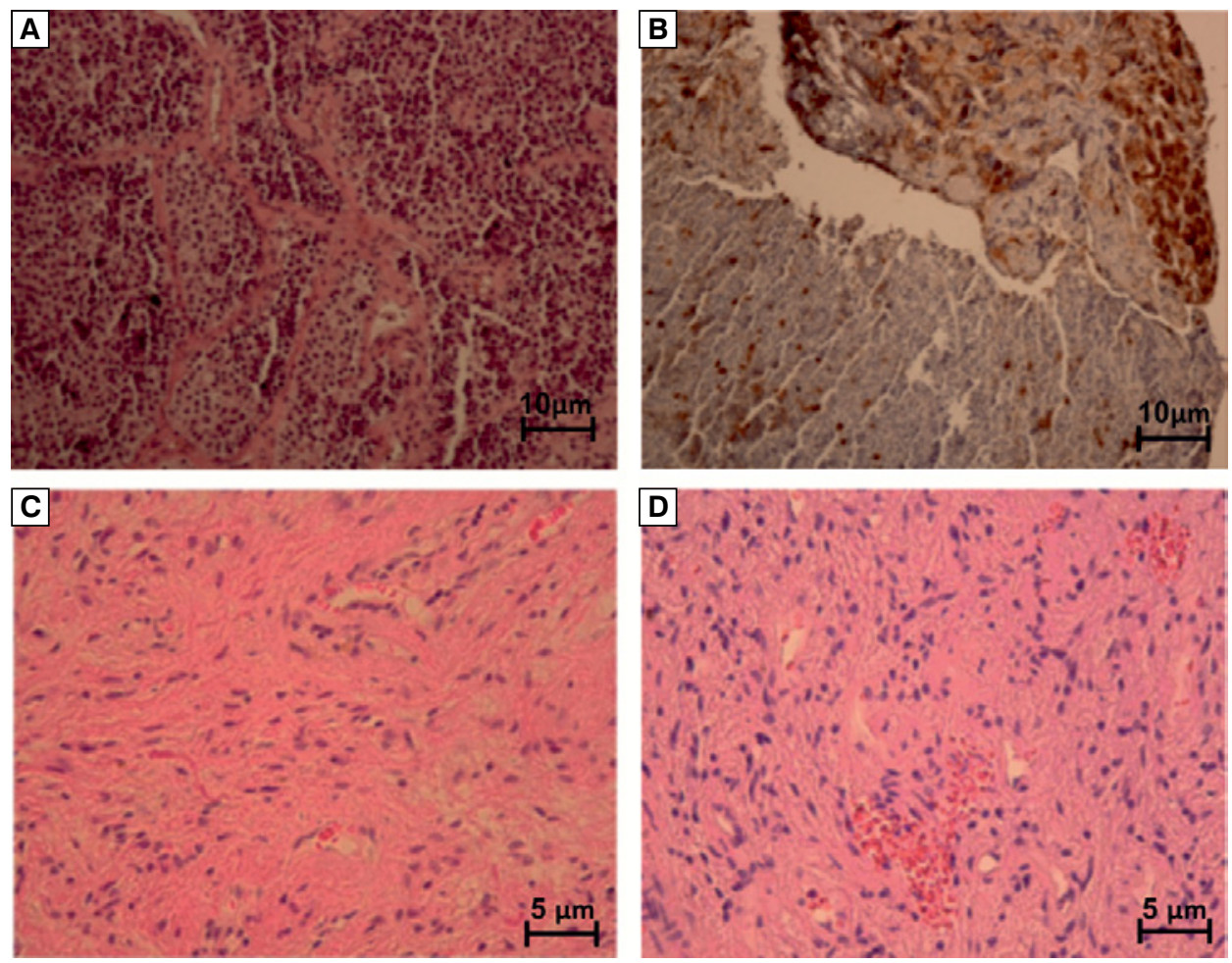

Figure 2A. Pseudolobular structure of the adenoma imitating normal adenopituitary structure; B. Pituitary adenoma. Staining for ACTH. Normal adenohypophisis positive for ACTH is seen; C. Pilocytic astrocytoma; D. Normal adenohypophysis.

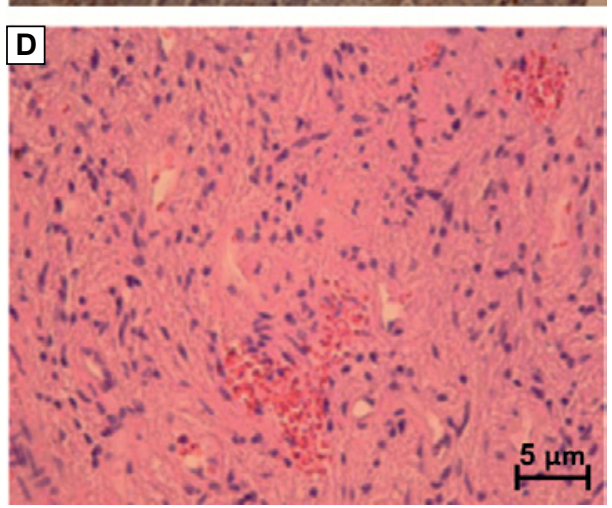

for the histopathological investigation. However, it is not always the rule, because it may be influenced by different factors. Contemporary art of pituitary tumor surgery leads to minimization of the surgical approach and obtaining small amount of material for histopathological examination. The pituitary adenomas are usually easily removed during the surgery due to their soft, often semi-fluid consistency (Fig. 1). On the other side, it may determine difficulties while collecting the tumor mass for histopathological investigation, because it can be inadvertently aspirated. Because of the small volume, a sample run dry quickly, therefore an immediate transfer to the neuropathological lab is crucial.

An appropriate interpretation of the histopathological examination might be impaired by the presence of the regular components of the pituitary gland (adenohypophysis, neurohypophysis), other tissue elements and additional pathological findings (Fig. 2B). Commonly encountered are bleeding traces (siderophags, hemosiderin and hematin deposits), less often cholesterol granuloma, amyloid deposits (in case of some prolactinoma and somatotropinoma), calcifications (mainly prolactinoma). [9,14-15] Differentiating between adenohypophysis and the adenoma might be difficult even for an experienced neuropathologist, particularly when adenoma is accompanied by hyperplastic cells (mainly corticotrophs and mammotrophs) [16]. In a number of cases, tissue of neurohypophysis is taken together with 
the tissue of adenoma. It may be mistaken with pilocytic astrocytoma because of it morphological similarity. (Fig. 2C and 2D)

From the neurosurgical perspective, it is important to confirm the diagnosis of pituitary adenoma. In turn for a neuropathologist adequate amount of material provides possibility of bright spectrum of histopathological analysis including the panel of immunohistochemical staining.

We aimed to determine the frequency of unreliable material for histopathological examination and factors influencing the reliability of histopathological specimens after surgery for pituitary adenomas. The lack of sufficient knowledge in that field and discrepancies in the literature explain the purpose of the study.

\section{Material and methods}

We enrolled patients operated in the Department of Neurosurgery and subsequently diagnosed in the Department of Neuropathology during ten year-period (2007-2016). The final study group consisted of 72 ( 44 females) patients aged between 10 and 84 years (mean age: 46.9 years) with complete clinical, histopathological and radiological records. All patients were operated microsurgically through the sphenoid sinus. Specimens were embedded in paraffin. Then, in all cases routine H\&E staining was carried out alongside with full panel of immunohistochemical staining for hormones of the adenohypophysis, such as: prolactine (anti-PRL, Dako, Denmark), growth hormone (anti-GH, Dako, Denmark), adronocorticothropic hormone (anti-ACTH, Dako, Denmark), thyreotropic hormone (anti-TSH, Novocastra, Great Brittan), lutethropic and follicular hormones (anti-LH and anti-FSH, Dako, Denmark and $\alpha$-subunite (anti- $\alpha$-SU, Novocastra, Great Brittan). All sections were examined using light microscope Nikon Optishot-2 under magnification of 200x and 400x.

Radiological analysis of the tumor was based on magnetic resonance imaging of the sellar region. The tumor volume was calculated using the Di Chiro and Nelson formula $V=(\varpi / 6) \times(x \times y \times z)$ and was given in cubic millimeters $\left(\mathrm{mm}^{3}\right)$ [17].

Area of specimen was calculated using ImageJ software v. $1.43 \mathrm{k}$ (National Institute of Health, USA). In cases of specimen fragmentation the areas of fragments were calculated separately and added. Area of specimen is given in square millimeters $\left(\mathrm{mm}^{2}\right)$.

\section{Statistical analysis}

The results were presented using descriptive statistical methods, including ranges, means, standard devi- ations, and percentage distributions. Continuous variables were assessed using the t-test or Mann-Whitney $\mathrm{U}$ test when appropriate. Proportions were assessed using the Pearson's $\chi^{2}$ test. The Pearson's correlation coefficient was used to establish the relation between volume of the adenoma and area of a histopathological specimen.

Statistical analyses were performed using Statistica 9.0 PL, (StatSoft, Poland).

\section{Results}

Five hundred and four immunohistochemically stained specimens derived from 72 patients were analyzed. Eighty four specimens were positive for adenoma tissue. We observed one or more positive immunohistochemical reactions in 43 patients. We diagnosed 22 monohormonal (30.56\%) 21 plurihormonal $(29.17 \%)$ and $21(29.17 \%)$ adenomas with negative hormonal staining. In 8 cases $(11.11 \%)$, we were not able to interpret immunohistochemical staining. Therefore, in these cases, we recognized specimen as unreliable. Macroadenomas and microadenomas were diagnosed in 51 (70.84\%) and 21 (29.16\%) cases, respectively.

Meantumorvolumewas6405.01 $\pm 12068.27 \mathrm{~mm}^{3}$. Mean area of specimen was $41.79 \pm 37.79 \mathrm{~mm}^{2}$. Association between area of specimen and tumor volume was positive when tested with the Pearson's correlation coefficient $(r=0.496, p<0.001)$ - Fig. 3 . There was no correlation between unreliable specimen and tumor volume. Unreliable specimens had significantly smaller area (Table I).

The most frequent incidental finding in specimen was adenohypophisis fragments, found in 29 cases (40.28\%). In unreliable specimen, there was a higher incidence of hemorrhage $(75.00 \%$ vs. $21.88 \% ; p=0.002)$,

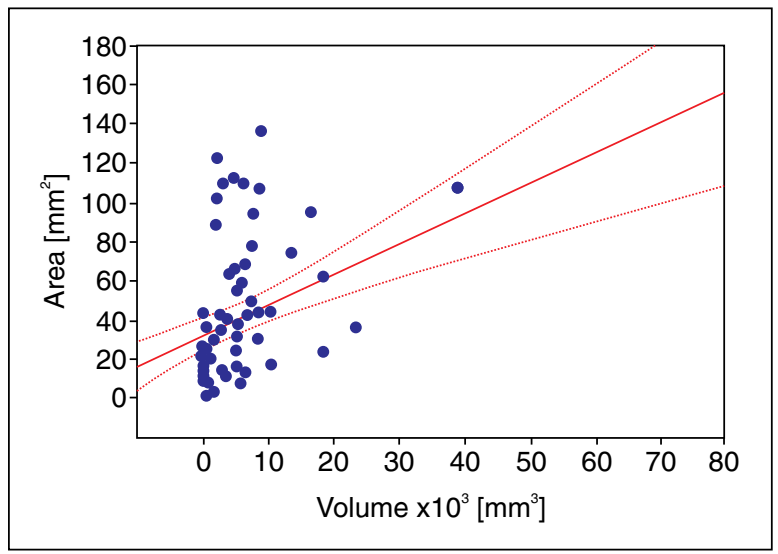

Figure 3. The relationship between the histopathological specimen area and the tumor volume 
Table 1. The tumor volume based on MRI and H\&E specimen area in the groups of reliable and unreliable specimens

\begin{tabular}{|c|c|c|c|c|c|c|c|c|c|c|c|}
\hline & \multicolumn{5}{|c|}{ Unreliable } & \multicolumn{5}{|c|}{ Reliable } & \multirow{2}{*}{$p$ value } \\
\hline & $\mathbf{n}$ & Av. & SD & Min. & Max. & $\mathbf{n}$ & Av. & SD & Min. & Max. & \\
\hline Volume $\left(\mathrm{mm}^{3}\right)$ & 8 & 729.41 & 978.12 & 18.8 & 2825 & 64 & 7114.46 & 12626.54 & 6.2 & 70475 & $p=0.159$ \\
\hline Area $\left(\mathrm{mm}^{2}\right)$ & 8 & 14.41 & 14.01 & 0.55 & 36.71 & 64 & 45.22 & 38.48 & 1.22 & 156.15 & $p=0.028$ \\
\hline
\end{tabular}

Table 2. The reliability of the specimens and additional findings

\begin{tabular}{|c|c|c|c|c|c|}
\hline \multirow[t]{2}{*}{ Incidental findings } & \multicolumn{2}{|c|}{ Unreliable $(n=8)$} & \multicolumn{3}{|c|}{ Reliable $(n=64)$} \\
\hline & $\mathbf{n}$ & $\%$ & $\mathbf{n}$ & $\%$ & $p$ value \\
\hline Adenohypophysis & 3 & 37.50 & 26 & 40.62 & NS \\
\hline Hemorrhage & 6 & 75.00 & 14 & 21.88 & $p=0,002$ \\
\hline Neurohypophysis & 3 & 37.50 & 2 & 3.13 & $p=0,004$ \\
\hline Necrosis & 2 & 25.00 & 1 & 1.56 & $p=0,002$ \\
\hline Thermal artefacts & 1 & 12.50 & 2 & 3.13 & NS \\
\hline Inflammatory changes & 0 & 0 & 2 & 3.13 & NS \\
\hline Fibrosis & 0 & 0 & 2 & 3.13 & NS \\
\hline Hyalinisation & 0 & 0 & 2 & 3.13 & NS \\
\hline Neural tissue of the hypothalamus & 0 & 0 & 1 & 1.56 & NS \\
\hline Respiratory epithelium & 0 & 0 & 1 & 1.56 & NS \\
\hline Vessels & 0 & 0 & 1 & 1.56 & NS \\
\hline Cholesterol granuloma & 0 & 0 & 1 & 1.56 & NS \\
\hline Dried material & 1 & 12.50 & 0 & 0 & NS \\
\hline
\end{tabular}

neurohypophysis tissue (37.50\% vs. 3.13\%; $p=0.004)$ and necrosis (25.00\% vs. $1.56 \% ; p=0.002)$ (Table II).

\section{Discussion}

In our study, we established the incidence of unreliable histopathological specimen of pituitary adenomas at the level of $11.11 \%$. Moreover, we found a significant correlation between the area of specimen and its reliability, but there is no correlation between the tumor volume and the reliability of the specimen. The factors most frequently associated with specimens unreliability included: hemorrhage, incidental neurohypophysis tissue and necrosis. Those additional findings play a role especially in case of small area specimens, where available field for histopathological examination was critically reduced.

Histopathological diagnosis of pituitary adenomas is based on routine histopathological staining as well as immunoexpression of the pituitary hormones [18]. The World Health Organization (WHO) standards despite thorough description of the diagnostic criteria for pituitary adenoma are mute in case of unreliable material [19-20]. Therefore it is a surgeon's duty to preserve the reliable specimen to allow the accurate diagnosis while removing adenoma. This is dependent on a number of circumstances such as the size and consistence of the tumor, bleeding during surgery and handling with the specimen after its removal.

The relation between the available histopathological material and tumor size was investigated. The relation of the adenoma volume (according to the MRI picture) and specimen area was recognized as a reliability parameter. The strong correlation between those two parameters was found ( $p<0.001$ ). The statistical analysis showed clearly that the greater the volume of the tumor, the greater amount of collected histopathological material. On the other hand, analyzing the diagram that shows the relation between adenomas volume and area of the specimens, the curve trend exist through few points corresponding to the large tumors. However most of the points were focused in the portion of the diagram corresponding to small and medium tumors. Tumors that did not differ much in size are represented by specimens of huge spread of area (Fig. 3). It seemed that it bared witness to a great impact of the neurosurgeon and intraoperative conditions on the amount of collected histopathological material. It is necessary to excuse the surgeon in case of very small 
tumors, where securing of the histopathological material may be difficult. Notwithstanding it is not possible to define the smallest size of the adenoma with reliable histopathological material, because even based on very small tumors it is possible to prepare conclusive specimens.

The previous WHO classification mentioned that only a small number of pituitary tumors remained undiagnosed and categorized as unclassified adenomas [21]. This fact is mentioned by Saeger et al. [6]. That categorization is not followed in the 2017 classification [19]. This study found that pituitary adenomas recognized as unclassified are more frequently diagnosed based on small specimen with higher incidence of necrosis and atypical structures. Other authors mentioned only unreliable specimens and usually exclude them from the study group [16]. Knosp et al. also acknowledge the fact that small specimen may not be representative enough for accurate immunohistochemical staining and accurate diagnosis [22].

Overall unreliability of histopathological specimen in our study was established a little over $11 \%$. This frequency is similar to one described by Saeger et al, which was $8 \%$ [23]. In study by Maksymowicz et al., unreliability was established at $16.3 \%$ and was mainly associated with small specimens size and presence of normal pituitary tissue admixture [16].

The awareness of an operating neurosurgeon of the importance of meticulous collecting histopathological material, especially in microadenoma cases, has essential impact on further neuropathological evaluation and possibility of immunohistochemical staining.

In conclusion, the presence of some additional tissue elements and artifacts in the histopathological specimen makes the immunohistochemical evaluation difficult or even impossible. However, this problem relates to a relatively small percentage of cases, mainly small tumors. In those cases, the resignation of intraoperative histopathological investigation should be considered.

\section{References}

1. Arafah BM, Nasrallah MP. Pituitary tumors: pathophysiology, clinical manifestations and management. Endocr Relat Cancer. 2001; 8(4): 287-305, indexed in Pubmed: 11733226.

2. Casanueva FF, Molitch ME, Schlechte JA, et al. Guidelines of the Pituitary Society for the diagnosis and management of prolactinomas. Clin Endocrinol (Oxf). 2006; 65(2): 265-273, doi: 10.1111/j.1365-2265.2006.02562.x, indexed in Pubmed: 16886971

3. Gsponer J, De Tribolet N, Déruaz JP, et al. Diagnosis, treatment, and outcome of pituitary tumors and other abnormal intrasellar masses. Re- trospective analysis of 353 patients. Medicine (Baltimore). 1999; 78(4): 236-269, indexed in Pubmed: 10424206.

4. Dorn A, Nyström S, Müller M, et al. Immunocytochemical characterisation of human pituitary adenomas. Zentralbl Neurochir. 1985; 46(3): 188-194, indexed in Pubmed: 3911665

5. Kleinschmidt-DeMasters BK. Subtyping does matter in pituitary adenomas. Acta Neuropathol. 2006; 111(1): 84-85, doi: 10.1007/s00401005-1105-6, indexed in Pubmed: 16328517

6. Nosé V, Grossman A, Mete O. Lactotroph adenoma. In: Lloyd RV, Osamura RY, Klöppel G, Rosai J, editors. WHO Classification of Tumours of Endocrine Organs, 4th edition. IARC Press, Lyon. : 2017: 24-27.

7. Hamid Z, Mrak R, ljaz M, et al. Sensitivity and Specificity of Immunohistochemistry in Pituitary Adenomas. The Endocrinologist. 2009; 19(1): 38-43, doi: 10.1097/ten.0b013e31819390ce.

8. Kontogeorgos G. Predictive markers of pituitary adenoma behavior. Neuroendocrinology. 2006; 83(3-4): 179-188, doi: 10.1159/000095526, indexed in Pubmed: 17047381.

9. Horvath E, Scheithauer B, Kovacs K, et al. Hypothalamus and pituitary. In: Graham D, Lantos P, editors. Greenfield's Neuropathology. 7th edition. Edward Arnold, Londyn. ; 2000: 983-1062.

10. Kontogeorgos G, Kovacs K, Lloyd RV, et al. Plurihormonal and double adenomas. In:, Osamura RY, Klöppel G, Rosai J, editors. WHO Classification of Tumours of Endocrine Organs. 4th edition. IARC Press, Lyon. ; 2017: 39-40.

11. Pawlikowski M, Kunert-Radek J, Radek M. Plurihormonality of pituitary adenomas in light of immunohistochemical studies. Endokrynol Pol. 2010; 61 (1): 63-66, indexed in Pubmed: 20205106.

12. Saeger W, Lüdecke DK, Buchfelder M, et al. Pathohistological classification of pituitary tumors: 10 years of experience with the German Pituitary Tumor Registry. Eur J Endocrinol. 2007; 156(2): 203-216, doi 10.1530/eje.1.02326, indexed in Pubmed: 17287410

13. Sano T. Comments on the WHO histological classification of pituitary tumors. Acta Neuropathol. 2006; 111(1): 82-83, doi: 10.1007/s00401005-1104-7, indexed in Pubmed: 16317556

14. Matsuo T, Mori H, Nishimura Y, et al. Quantification of immunohistochemistry using an image analyser: correlation with hormone concentrations in pituitary adenomas. Histochem J. 1995; 27(12): 989-996, indexed in Pubmed: 8789400.

15. Scheithauer B, Kovacs K, Horvath E, et al. Pathology of the Pituitary and Sellar Region. Practical Surgical Neuropathology. 2010: 371-416, doi: 10.1016/b978-0-443-06982-6.00018-3.

16. Maksymowicz M. Ocena przydatności mikroskopii elektronowej i immunocytochemii na poziomie ultrastrukturalnym w diagnostyce klasyfikacji gruczolaków przysadki [dissertation]. Medical University of Warsaw. ; 2002

17. DI CHIRO G, NELSON KB. The volume of the sella turcica. Am J Roentgenol Radium Ther Nucl Med. 1962; 87: 989-1008, indexed in Pubmed: 13885978

18. Yamada S, Ohyama K, Taguchi M, et al. A study of the correlation between morphological findings and biological activities in clinically nonfunctioning pituitary adenomas. Neurosurgery. 2007: 61(3): 580-4: discussion 584, doi: 10.1227/01.NEU.0000290906.53685.79, indexed in Pubmed: 17881972.

19. Osamura RY, Grossman A, Korbonits M, et al. Pituitary adenoma. In: Lloyd RV, , Klöppel G, Rosai J, editors. WHO Classification of Tumours of Endocrine Organs. 4th edition. IARC Press, Lyon. ; 2017: 13.

20. Perry A, Scheithauer BW. Commentary: Classification and grading of pituitary tumors. Observations of two working neuropathologists. Acta Neuropathol. 2006; 111(1): 68-70, doi: 10.1007/s00401-005-1098-1, indexed in Pubmed: 16328523

21. Lloyd RV, Kovacs K, Young Jr. Pituitary tumors: Introduction. In: Delellis RA, , Heitz PU, Eng C, editors. World Health Organisation Classification of Tumours: Pathology \& Genetics - Tumours of Endocrine Organs. Lyon: IARC Press. ; 2004: 10-13.

22. Knosp E Krisch K Schmidbauer M et al. [Immunologic hormone detection in hypophyseal adenomas: correlation of serum hormone findings with immunocytochemical hormone levels in tumor tissue] Wien Klin Wochenschr. 1988; 100(10): 322-325, indexed in Pubmed: 2840774.

23. Saeger W. Current pathological classification of pituitary adenomas. Acta Neurochir Suppl. 1996; 65(2): 1-3, indexed in Pubmed: 8738481. 